\title{
Multi-species simulation of Trichel pulses in oxygen
}

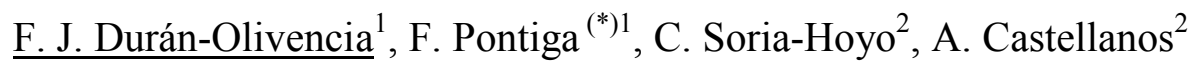 \\ ${ }^{l}$ Dpt. Física Aplicada II, ETS Ingeniería de Edificación,Universidad de Sevilla, Av. Reina Mercedes $4 a$, \\ 41012 Sevilla, Spain \\ ${ }^{3}$ Dpt. Electrónica y Electromagnetismo, Facultad de Física, Universidad de Sevilla, Av. Reina Mercedes s/n, \\ 41012 Sevilla, Spain \\ ${ }^{(*)}$ pontiga@us.es
}

\begin{abstract}
A multi-species model consisting of seven species has been implemented to simulate the generation and development of Trichel pulses in oxygen between a sphere (the cathode) and a plane (the anode). The spatial and temporal evolution of species is obtained by solving the continuity equations of species using a classical one-dimensional model of negative corona discharge. The chemical kinetics of corona discharge includes electron impact reactions (ionization, dissociative and non-dissociative electron attachment, molecular dissociation, etc.), charge transfer reactions and reactions between neutral species.
\end{abstract}

Trichel pulses occur in electronegative gases when the applied voltage is slightly increased from the threshold value of corona discharge. These pulses are characterized by a short duration, of the order of tens or hundreds of nanoseconds, followed by a longer inter-pulse period of tens of microseconds. The concurrence of different temporal scales makes the numerical simulation of Trichel pulses a challenging task, which has been dealt by different investigators using $1 \mathrm{D}$ and $2 \mathrm{D}$ numerical simulations [1-5]. Usually, only three generic species are considered in these studies: electrons, positive ions and negative ions. Therefore, the plasma kinetics of the corona discharge is simplified to the fundamental processes of ionization, electron attachment and recombination.

In this work, the generation of Trichel pulses will be simulated using the classical 1D model developed by Morrow [1], but it will be extended to include electrons, four different type of ions $\left(\mathrm{O}_{2}{ }^{+}\right.$, $\mathrm{O}_{2}{ }^{-}, \mathrm{O}_{3}{ }^{-}$and $\left.\mathrm{O}^{-}\right)$, three neutral species $\left(\mathrm{O}_{2}, \mathrm{O}_{3}\right.$ and $\left.\mathrm{O}\right)$ and 14 chemical reactions between these species. The electrical discharge is assumed to occur in low pressure oxygen (50 Torr), between a sphere, with radius $R=2 \mathrm{~mm}$, and a grounded plane at a distance $d=20 \mathrm{~mm}$. The sphere is subjected to a negative high voltage, and the discharge channel is modeled as a cylinder with radius $r=0.8 \mathrm{~mm}$, centered along the axis of symmetry and having a uniform radial distribution of species, but variable

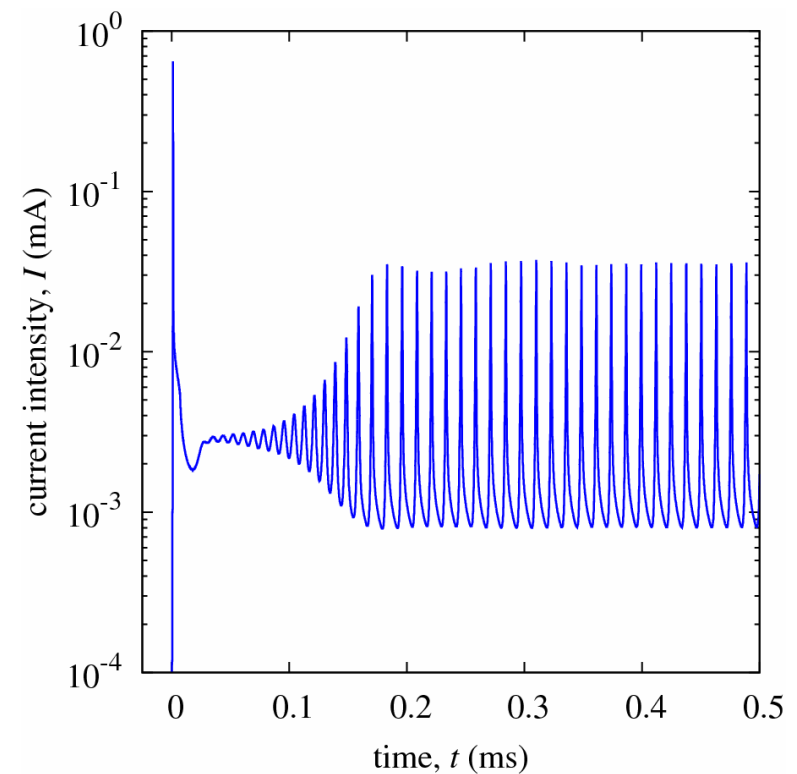

Fig. 1: Current intensity versus time corresponding to an applied voltage of $V=1750 \mathrm{~V}$.

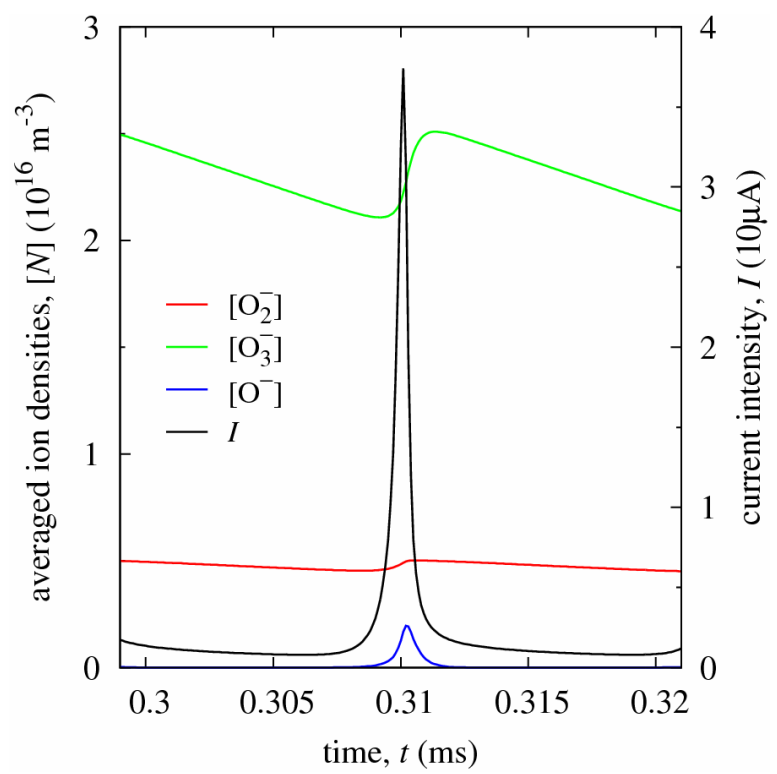

Fig. 2: Temporal evolution of the spatial average of the ion densities (left axis) and current intensity (right axis) in a single Trichel pulse $(V=1750 \mathrm{~V})$. 


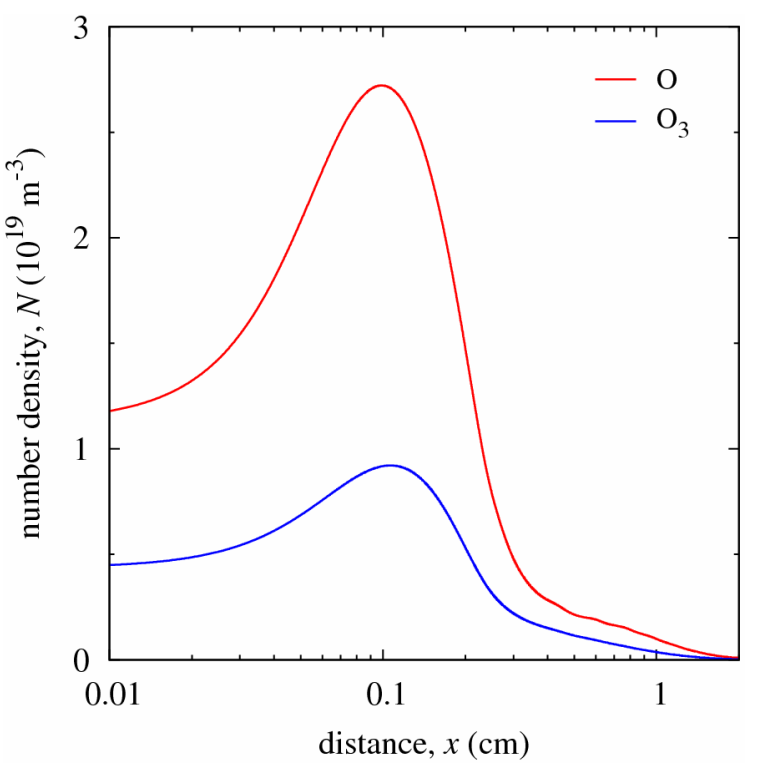

Fig. 3: Spatial distribution of atomic oxygen and ozone densities correspoding to $t=0.41 \mathrm{~ms}$ and $V=1750 \mathrm{~V}$.

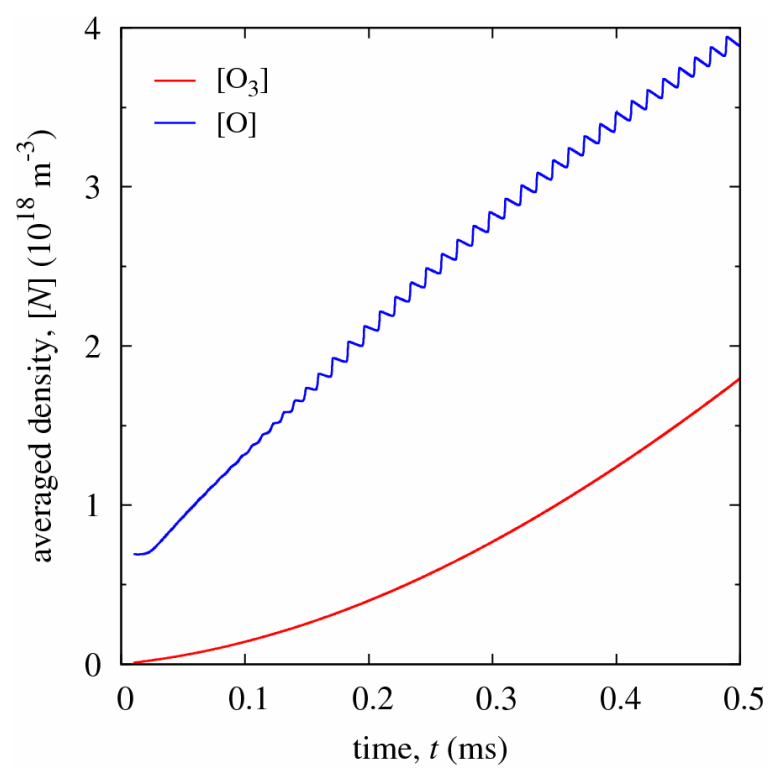

Fig. 4: Temporal variation of the spatial average of atomic oxygen and ozone densities for $V=1750 \mathrm{~V}$.

in the axial direction. A finite-element-method based solver was used to deal with the computational task of numerically integrating the continuity equation for each species and Gauss law for the electric field. The reactions included in the chemical kinetics model and their corresponding reaction rate constants were taken from Eliasson and Kogelschatz's report [6] (reaction numbers: 1, 2, 4, 7a, 9a, 11, 26a, 26b, 28a, 28b, 36a, 49, 51 and 52).

Figure 1 shows the temporal evolution of the current intensity for an applied voltage of $1750 \mathrm{~V}$. Clearly, after an initial pulse which develops in a space charge free gap, the corona discharge enters in a stable pulsating regime with a frequency of about $78 \mathrm{kHz}$. The temporal evolution of the negative ion densities (spatially averaged) along a regular Trichel pulse is depicted in figure 2 . The negative space charge is mainly constituted by ozone ions, $\mathrm{O}_{3}{ }^{-}$, and, in a lesser degree, by oxygen ions, $\mathrm{O}_{2}{ }^{-}$. In contrast, atomic oxygen ions, $\mathrm{O}^{-}$, are quickly converted into $\mathrm{O}_{3}{ }^{-}$and $\mathrm{O}_{2}{ }^{-}$through fast charge transfer reactions, and their existence is limited to the duration of the Trichel pulse.

Regarding the density of neutral species, the spatial distribution of atomic oxygen and ozone are presented in figure 3 after $0.41 \mathrm{~ms}$ of the initiation of the discharge. Atomic oxygen is mainly generated by electron impact dissociation of oxygen molecules, and ozone is then produced by the association of atomic and molecular oxygen in a three body reaction. The generation of $\mathrm{O}$ requires energetic electrons capable of breaking oxygen molecules, which are produced at a certain stage of each Trichel pulse. Consequently, the spatially averaged number density of atomic oxygen exhibits a stair-like behavior with time, due to its correlation with the triggering of Trichel pulses (figure 4). On the contrary, ozone has a long life-time, and its number density increases steadily with time at an approximate rate of $6 \times 10^{16}$ molecules $/ \mathrm{m}^{-3}$ per Trichel pulse $\left(\sim 2.4 \times 10^{-3} \mathrm{ppm} / \mathrm{pulse}\right)$.

\section{Acknowledgements}

This work was supported by the "Consejeria de Innovacion, Ciencia y Empresa (Junta de Andalucia)" within the European Regional Development Fund contract FQM-4983, and by the Spanish Government Agency "Ministerio de Ciencia e Innovacion" (contract FIS2011-25161).

\section{References}

[1] R. Morrow, Phys. Rev. A 32 (1985) 1799-809.

[2] A. P. Napartovich, Y. S. Akishev, A. A. Deryugin, I. V. Kochetov, M. V. Pankin, J. Phys. D: Appl. Phys. 30 (1997) 2726-36.

[3] C. Soria-Hoyo, F. Pontiga, A. Castellanos, J. Phys. D: Appl. Phys. 40 (2007) 4552-4560.

[4] P. Sattari, C. F. Gallo, G. S. P. Castle, K. Adamiak, J. Phys. D: Appl. Phys. 44 (2011) 155502 (11pp).

[5] T. N. Tran, I. O. Golosnoy, P. L. Lewin, G. E. Georghiou, J. Phys. D: Appl. Phys. 44 (2011) 015203 (15pp).

[6] B. Eliasson and U. Kogelschatz, Technical Report KLR 86-11 C (1986), Brown Boveri. 